\title{
Nanotheranostics
}

2022; 6(1): 91-102. doi: 10.7150/ntno.63124

Review

\section{Near-infrared probes for luminescence lifetime imaging}

\author{
Benhao Li1 $1,2,3,4$, Jing Lin ${ }^{1}$, Peng Huang ${ }^{1 凶}$, Xiaoyuan Chen ${ }^{2,3,4}$ \\ 1. Marshall Laboratory of Biomedical Engineering, International Cancer Center, Laboratory of Evolutionary Theranostics (LET), School of Biomedical \\ Engineering, Shenzhen University Health Science Center, Shenzhen 518060, China \\ 2. Departments of Diagnostic Radiology, Surgery, Chemical and Biomolecular Engineering, and Biomedical Engineering, Yong Loo Lin School of Medicine \\ and Faculty of Engineering, National University of Singapore, Singapore, 119074, Singapore \\ 3. Clinical Imaging Research Centre, Centre for Translational Medicine, Yong Loo Lin School of Medicine, National University of Singapore, Singapore 117599, \\ Singapore \\ 4. Nanomedicine Translational Research Program, NUS Center for Nanomedicine, Yong Loo Lin School of Medicine, National University of Singapore, \\ Singapore 117597, Singapore \\ In memory of Dr. Sanjiv Sam Gambhir and Dr. Moritz Kircher.
}

$\bowtie$ Corresponding authors: Peng Huang, PhD, E-mail: peng.huang@szu.edu.cn; Xiaoyuan (Shawn) Chen, PhD, E-mail: chen.shawn@nus.edu.sg

(c) The author(s). This is an open access article distributed under the terms of the Creative Commons Attribution License (https://creativecommons.org/licenses/by/4.0/). See http://ivyspring.com/terms for full terms and conditions.

Received: 2021.05.25; Accepted: 2021.06.29; Published: 2022.01.01

\begin{abstract}
Biomedical luminescence imaging in the near-infrared (NIR, 700-1700 nm) region has shown great potential in visualizing biological processes and pathological conditions at cellular and animal levels, owing to the reduced tissue absorption and scattering compared to light in the visible $(400-700 \mathrm{~nm})$ region. To overcome the background interference and signal attenuation during intensity-based luminescence imaging, lifetime imaging has demonstrated a reliable imaging modality complementary to intensity measurement. Several selective or environment-responsive probes have been successfully developed for luminescence lifetime imaging and multiplex detection. This review summarizes recent advances in the application of luminescence lifetime imaging at cellular and animal levels in NIR-I and NIR-II regions. Finally, the challenges and further directions of luminescence lifetime imaging are also discussed.
\end{abstract}

Key words: Near-infrared probes, luminescence imaging, lifetime imaging, stimuli responsive, second near-infrared window

\section{Introduction}

Biomedical luminescence imaging has broad applications in life science due to its ability to achieve real-time investigation of physiological and pathological processes with fast feedback, high sensitivity, high spatiotemporal resolution [1-6]. In order to act as luminescence contrast agents, a lot of luminescence probes, including organic dyes [7-20], quantum dots (QDs) [21-25], rare-earth doped nanoparticles (RENPs) [26-31], single-walled carbon nanotubes (SWCNTs) [32,33], metal-ligand complexes [34,35] and semiconducting polymer nanomaterials (SPN) $[36,37]$ have been explored. Over the past few years, significant efforts have been made to create luminescence contrast agents that operate in the near-infrared window $(700-1700 \mathrm{~nm})$, which offers the enhanced tissue penetration over $5 \mathrm{~mm}$ [38-42]. Especially, luminescence imaging in the second near-infrared window (NIR-II, 1000-1700 nm), which is also called shortwave infrared region (SWIR), has shown higher resolution at deeper tissue penetration compared with NIR-I (700-1000 nm) window due to the reduced tissue scattering and diminished autofluorescence [43-54]. Meanwhile, recent works demonstrate that the autofluorescence of tissue becomes negligible for bioimaging beyond $1500 \mathrm{~nm}$ $[55,56]$. Unfortunately, only a few probes can achieve bioimaging beyond $1500 \mathrm{~nm}[57,58]$. As we know, accurate detection and bioimaging in deep tissue is still hampered by the fact that luminescence signal intensity exhibits unavoidable and inhomogeneous attenuation caused by tissue absorption and scattering [59-61]. Therefore, in vivo imaging of deep tissue requires the design and development of alternative techniques which is not based on the 
signal intensity.

Luminescence lifetime imaging has emerged as an important technique to detect or image specific biomarkers or physiological processes in living systems [62-64]. Luminescence lifetime is the reciprocal of rate constants of return routes from excited state to ground state [65]. The lifetime imaging requires imaging instrument to receive photons emitted from probes. Thus, the lifetime probes with long wavelength excitation and emission are capable of luminescence lifetime imaging in deep biological tissues. As for all luminescence parameters, the luminescence lifetime is the most direct insight into the interaction of contrast agents with their biological environment [66]. Lifetime imaging can report on physiological and pathological events that are difficult or impossible to detect through luminescence intensity imaging, because lifetime imaging is independent of contrast agent concentration and tissue penetration $[66,67]$. Compared with luminescence intensity, the luminescence lifetime provides a stable measurement method which is less susceptible to artifacts arising from tissue absorption and scattering, excitation intensity variations, probes photobleaching, or unknown tissue penetration depth [68].

\section{Luminescence lifetime imaging techniques}

Up to now, time-domain lifetime imaging techniques including time correlated single photon counting (TCSPC) and time-gated detections, and frequency-domain lifetime imaging technique, are two major ways to realize luminescence lifetime imaging. In time-domain fluorometry, the probes are excited by a pulsed light (Figure 1A). The width of pulsed light should be much shorter than the lifetime of the probes. After removing the excitation laser, the luminescence intensity of probes would decrease with the time. When the luminescence intensity decreases to the inverse of the natural base value e, the time duration required is called the luminescence lifetime $\tau$. In order to avoid the influence of anisotropy and rotational diffusion on the intensity decay, in general, the intensity decays are measured by a polarizer at $54.7 \mathrm{o}$ from the vertical $\mathrm{z}$-axis. For now, within time-domain lifetime imaging techniques, TCSPC and time-gated detections are widely to achieve lifetime imaging. The principle of TCSPC detection is shown in Figure 1B. After pulsed light excitation, less than one photon is detected per laser pulse. Photons are detected and recorded in the histogram. The $x$-axis is the time difference between the time of detected photons and paused pulsed laser. The y-axis is the number of detected photons during the time difference. Therefore, the histogram represents the waveform of the decay. For time-gated detection, the decay functions are measured by consecutive scanning and imaging for different delay of the gate pulse (Figure 1C). In the frequency-domain lifetime imaging technique, the probes are excited under intensity-modulated light, typically sine-wave modulation (Figure 1D). Generally, the modulation frequency of excitation light will reach over $100 \mathrm{MHz}$. When the probes are excited, the emissions are compelled to follow the same modulation frequency. The lifetime of probes comes from the time delay of emission relative to excitation. The delay time is measured according to the phase shift $(\Phi)$, based on the following formula, $\tau_{\Phi}=(1 / \omega) \tan \Phi, \quad(\omega$ : the modulation frequency in radians).
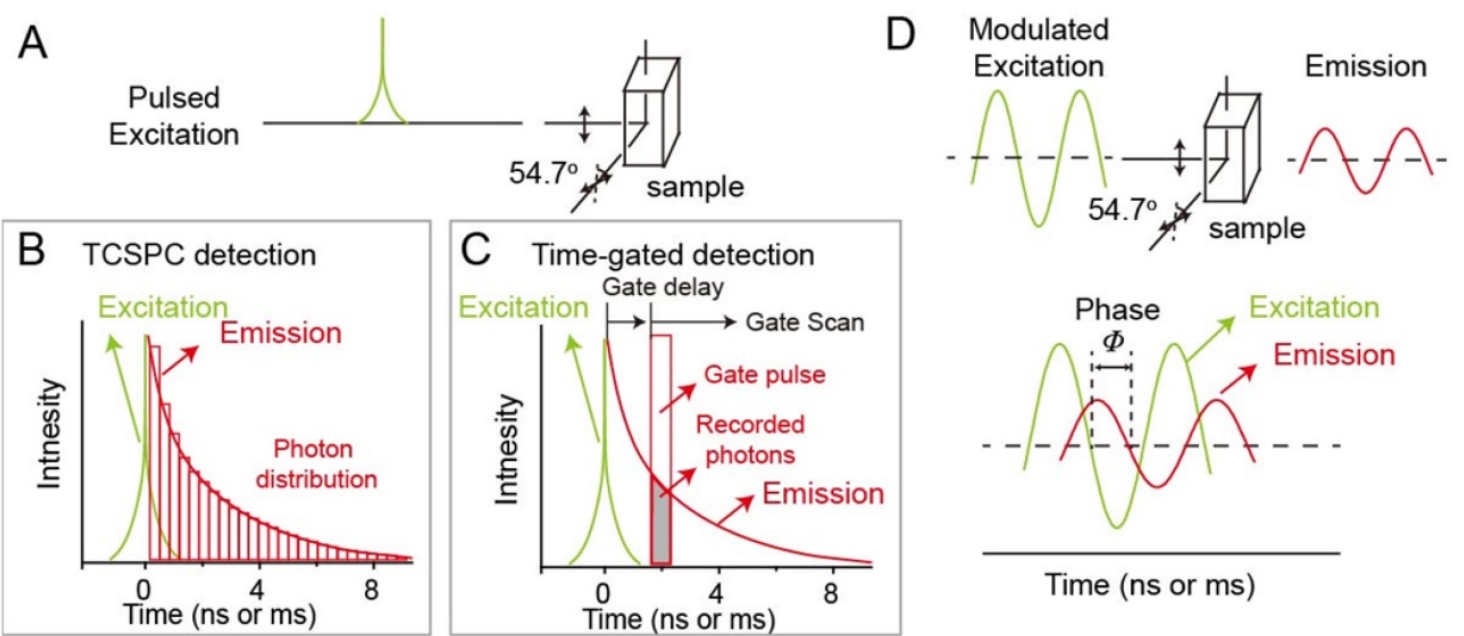

Time (ns or ms)

Figure 1. Luminescence lifetime imaging techniques. (A) Time-domain lifetime imaging techniques including time correlated single photon counting (TCSPC) detection (B) and time-gated detection (C). (D) Frequency-domain lifetime imaging technique. 
Table 1. Summary of NIR-I and NIR-II luminescence lifetime materials

\begin{tabular}{|c|c|c|c|c|c|c|}
\hline & Materials & Ex./Em. (nm) & Lifetime & $\begin{array}{l}\text { Designed } \\
\text { mechanism }\end{array}$ & Imaging applications & Ref. \\
\hline \multirow{3}{*}{$\begin{array}{l}\text { NIR-I } \\
\text { non-activatable } \\
\text { materials }\end{array}$} & cypate and DTTCI & $773 / 840$ & $0.5-1.1 \mathrm{~ns}$ & / & Multiple imaging & [82] \\
\hline & Yb-doped RENPs & $808 / 980$ & $0.7-1.3 \mathrm{~ms}$ & / & Multiple imaging & [83] \\
\hline & Tm-doped RENPs & $980 / 808$ & $78-2157 \mu \mathrm{s}$ & / & Multiple imaging & [84] \\
\hline \multirow[t]{8}{*}{$\begin{array}{l}\text { NIR-I activatable } \\
\text { materials }\end{array}$} & MMPSense750 FAST & $749 / 775$ & $0.63-0.76 \mathrm{~ns}$ & $\begin{array}{l}\text { Matrix metalloproteinase } \\
\text { activatable FRET probe }\end{array}$ & Imaging of breast cancer & [85] \\
\hline & PGC-800 & $778 / 794$ & $\begin{array}{l}0.29 \pm 0.01-0.47 \\
\pm 0.01 \text { ns. }\end{array}$ & $\begin{array}{l}\text { cathepsin B activatable FRET } \\
\text { probe }\end{array}$ & $\begin{array}{l}\text { Imaging of mouse infarcted } \\
\text { myocardium }\end{array}$ & [86] \\
\hline & CdTeSe/ZnS QDs and LS662 & $488 / 750$ & $\begin{array}{l}29 \text { ns at } \mathrm{pH}>7 \\
12 \text { ns at } \mathrm{pH}<5\end{array}$ & pH activatable FRET probe & / & [74] \\
\hline & Tm-doped RENPs and IR-820 & $785 / 800$ & $200-600 \mu \mathrm{s}$ & $\mathrm{ClO}^{-}$activatable FRET probe & Imaging of arthritis in mice & [87] \\
\hline & $\begin{array}{l}\text { Cypate and aliphatic polyester } \\
\text { dendrimer nanocomposites }\end{array}$ & $773 / 820$ & $0.36-0.98 \mathrm{~ns}$ & $\begin{array}{l}\mathrm{pH} \text {-activatable dye aggregation } \\
\text { state change }\end{array}$ & / & [76] \\
\hline & LS482 & $700 / 780$ & $1.16-1.4 \mathrm{~ns}$ & $\begin{array}{l}\mathrm{pH} \text {-induced dye protonated and } \\
\text { deprotonated form change }\end{array}$ & / & [88] \\
\hline & Palladium complex & $444 / 797$ & $50-300 \mu \mathrm{s}$ & Oxygen-induced NPs & $\begin{array}{l}\text { Imaging of oxygen } \\
\text { distribution and tissue } \\
\text { hypoxia }\end{array}$ & [89] \\
\hline & Pd-MX & $635 / 800$ & $75-270 \mu \mathrm{s}$ & Oxygen-induced NPs & $\begin{array}{l}\text { Imaging of tumor lesion in } \\
\text { mice liver }\end{array}$ & [90] \\
\hline $\begin{array}{l}\text { NIR-II } \\
\text { non-activatable } \\
\text { materials }\end{array}$ & $\begin{array}{l}\text { Nd, Ho, Pr, Tm and Er-doped } \\
\text { RENPs }\end{array}$ & $\begin{array}{l}808 / 1060(\mathrm{Nd}), 1155(\mathrm{Ho}), \\
1289 \text { (Pr), } 1475(\mathrm{Tm}), 1525 \\
(\mathrm{Er})\end{array}$ & $1.25-7.21 \mathrm{~ms}$ & / & Multiple imaging & {$[61]$} \\
\hline $\begin{array}{l}\text { NIR-II activatable } \\
\text { materials }\end{array}$ & DSNP@MY-1057-GPC-3 & $808 / 1060$ & $\begin{array}{l}203 \pm 2-298 \pm 2 \\
\text { Hs }\end{array}$ & ONOO-- activatable FRET probe & $\begin{array}{l}\text { Imaging of hepatocellular } \\
\text { carcinoma }\end{array}$ & [54] \\
\hline
\end{tabular}

Ex.: Excitation wavelength; Em.: Emission wavelength.

\section{Luminescence lifetime imaging applications in life sciences}

Compared to intensity measurements, luminescence lifetime imaging with higher reliability is widely used in life sciences, such as protein interactions [69-71], biologically relevant ions [72,73], $\mathrm{pH}$ [74-76], temperature $[77,78]$ etc. So far, luminescence lifetime imaging techniques have been used at the cellular level (luminescence lifetime imaging microscopy) [65,79-81] and at the animal level (wide-field luminescence lifetime imaging) [34,54,61]. Meanwhile, luminescence lifetime imaging has been carried out from visible to NIR-I and NIR-II. In this review, luminescence lifetime imaging in NIR-I and NIR-II regions are summarized in Table 1.

\section{Luminescence lifetime imaging in NIR-I region}

\section{Non-activatable luminescence lifetime imaging}

Luminescence probes which do not respond to stimuli in physiological conditions are called nonactivatable luminescence lifetime probes. Non-activatable luminescence lifetime probes have robust imaging signal and are widely used for multiplexing. Multiplex detection and imaging have shown great potential in nucleic acid analysis, protein profiling, and clinical diagnosis, which can simultaneously detect and identify multiple molecular species in one sample with minimal volume [91-93]. Optical multiplex imaging has become a promising method due to its advantages of flexibility, convenience, and fast feedback [94-96]. However, conventional multiplex imaging based on luminescence emission wavelength and intensity are limited to spectral overlap and background interference. Luminescence lifetime of probes is independent of intensity and therefore more tolerant to the ambient background. Thus, luminescence probes with different luminescence lifetimes can locate different biomolecules more accurately. Meanwhile, this unique method has great potential for imaging fluorophores with similar excitation/emission wavelengths and different luminescence lifetimes. Therefore, the color/lifetime binary strategy could exponentially scale the encoding capacity, unlocking the limitation of the channel number during multiplexing.

For example, Nothdurft and co-workers reported a home-made lifetime imaging system, including fiber coupled laser diode, a confocal laser scanning microscope, and TSCSPC card. This system can determine intracellular distributions of two NIR-I fluorescent dyes, including cypate and 3,3-diethylthiatricarbocyanine iodine (DTTCI), whose emission wavelengths are both located between 800 and $840 \mathrm{~nm}$ under $773 \mathrm{~nm}$ laser excitation (Figure 2 A, B) [82]. Luminescence intensity failed to achieve multiplex imaging due to the similar excitation and emission wavelengths of cypate and DTTCI. However, co-incubated NIR-I dyes could be easily distinguished and located due to the different lifetime values of 0.5 and $1.1 \mathrm{~ns}$ through lifetime imaging. This approach provides a tool for monitoring the distribution of dyes with similar excitation wavelength at the cellular level. Unfortunately, this imaging system cannot be applied at the animal level. To solve this issue, Ortgies and coworkers have developed time-gated technique based lifetime imaging system for in vivo multiplexed 
NIR-II lifetime imaging at the animal level. A series of $\mathrm{Yb}^{3+}$-doped RENPs were constructed via dopant engineering method with different lifetime (0.1-1.5 $\mathrm{ms})$. Luminescence lifetime imaging can distinguish the biological distribution of $\mathrm{NaY}_{0.6} \mathrm{Yb}_{0.1} \mathrm{Nd}_{0.3} \mathrm{~F}_{4} @ \mathrm{CaF}_{2}$ NPs and $\mathrm{NaY}_{0.7} \mathrm{Yb}_{0.1} \mathrm{Nd}_{0.2} \mathrm{~F}_{4} @ \mathrm{CaF}_{2}$ NPs with different lifetime times (0.7 and $1.3 \mathrm{~ms})$ after oral and intravenous administration in the same emission channel (Figure 2 C, D) [83]. Chen and coworkers developed Tm-doped upconversion nanoparticles with different lifetimes (from 78 to $2157 \mu$ s) and NIR-I luminescence at $808 \mathrm{~nm}$ under $980 \mathrm{~nm}$ laser (Figure 2 E). High contrast lifetime imaging in the liver and two subcutis can be achieved via intravenous and subcutaneous administration of NPs (Figure 2 F) [84]. Multiplexed lifetime imaging overcomes spectral overlap and background interference in wavelength/ intensity-dependent multiplex imaging. Therefore, it is a promising imaging modality for monitoring and tracking the distribution of biomolecules labelled with dyes or nanoparticles with different lifetimes to understand physiological or pathological processes.

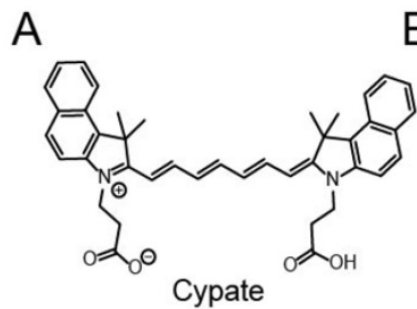

B Cypate alone

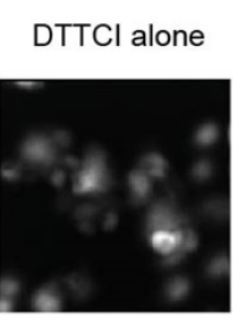

Both cypate

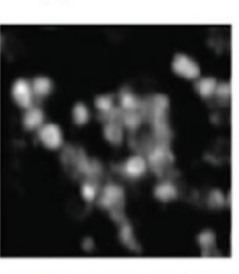

Ex/Em: $773 / 840 \mathrm{~nm}$

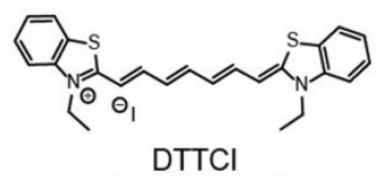

Ex/Em: $773 / 840 \mathrm{~nm}$
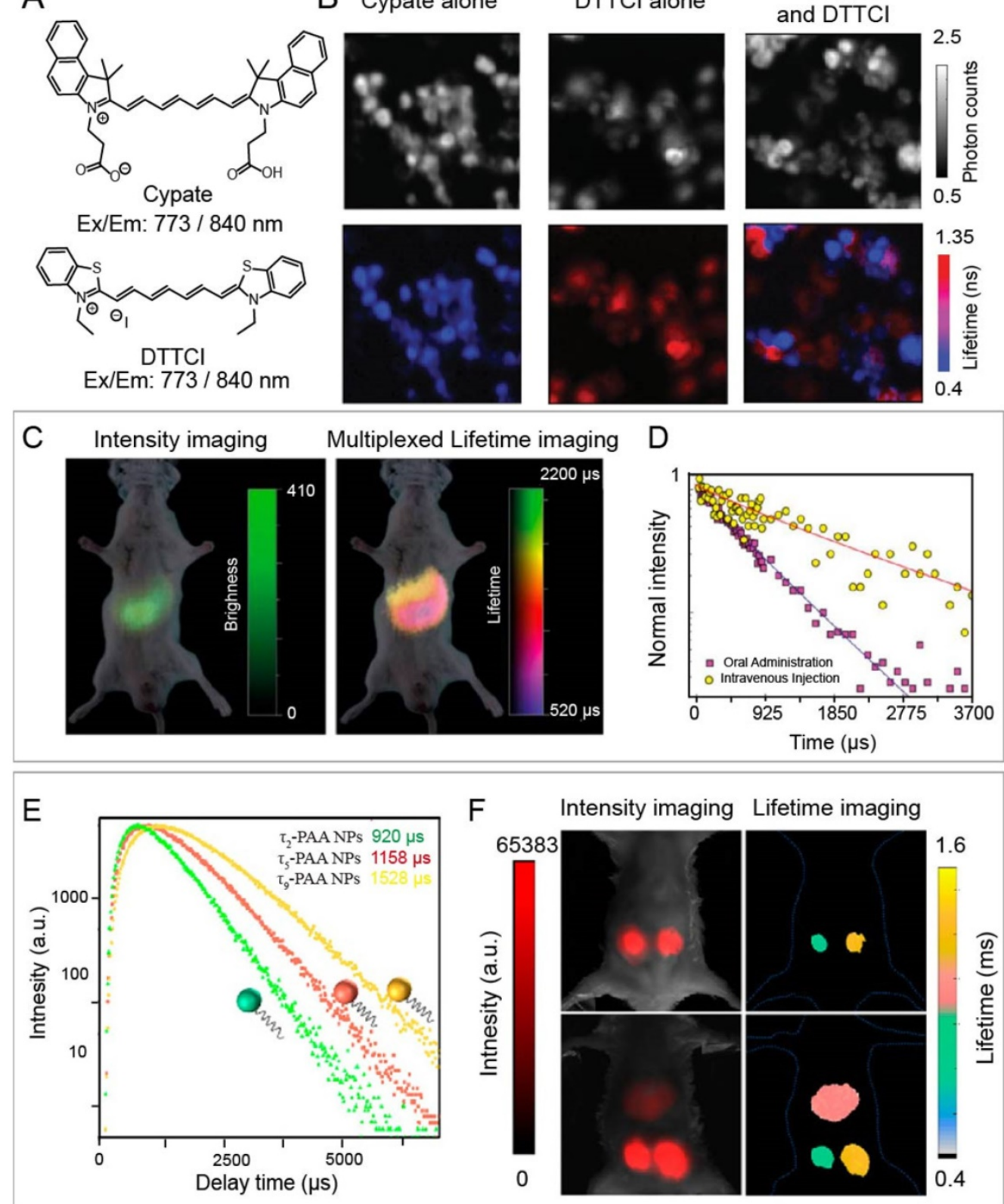

Figure 2. (A) Chemical structures of NIR-I dyes cypate and DTTCI. (B) luminescence intensity images (top row) and luminescence lifetime imaging microscopy images (bottom row) with cypate alone, with DTTCl alone, and with both cypate and DTTCl under $773 \mathrm{~nm}$ pulsed laser excitation. Adapted with permission from [82], copyright 2012. (C) Multiplexed intensity and lifetime images of healthy mice after oral and intravenous injection of Yb-doped RENPs. (D) Luminescence decay profile of the RENPs at two different locations. Adapted from permission from [83], copyright 2018. (E) Luminescence intensity decay of three poly(acrylic acid)-coated Tm-doped nanoparticles with different lifetimes of $\tau_{2}, \tau_{5}$, and $\tau_{9}$. (F) In vivo multiplexed lifetime imaging using three Tm-doped nanoparticles. Adapted with permission from [84], copyright 2020. 
A

Responsive Luminescence Lifetime based on FRET
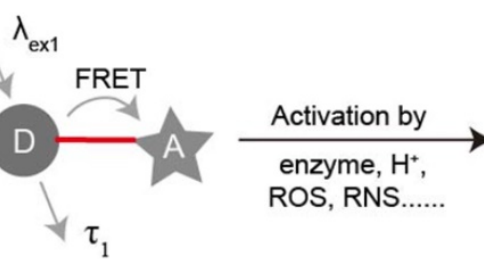

C

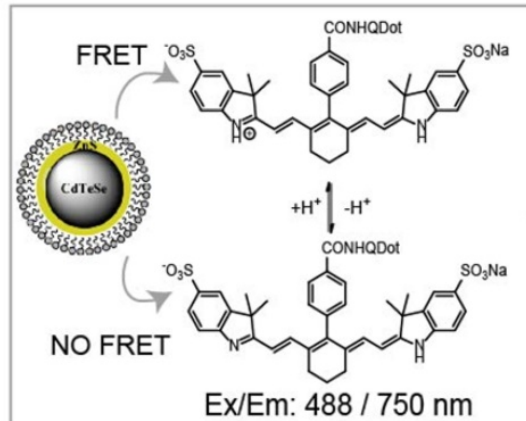

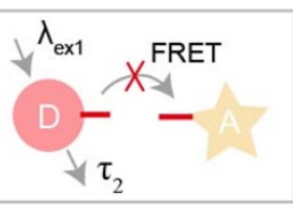
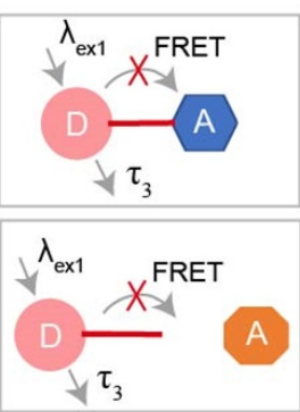

B
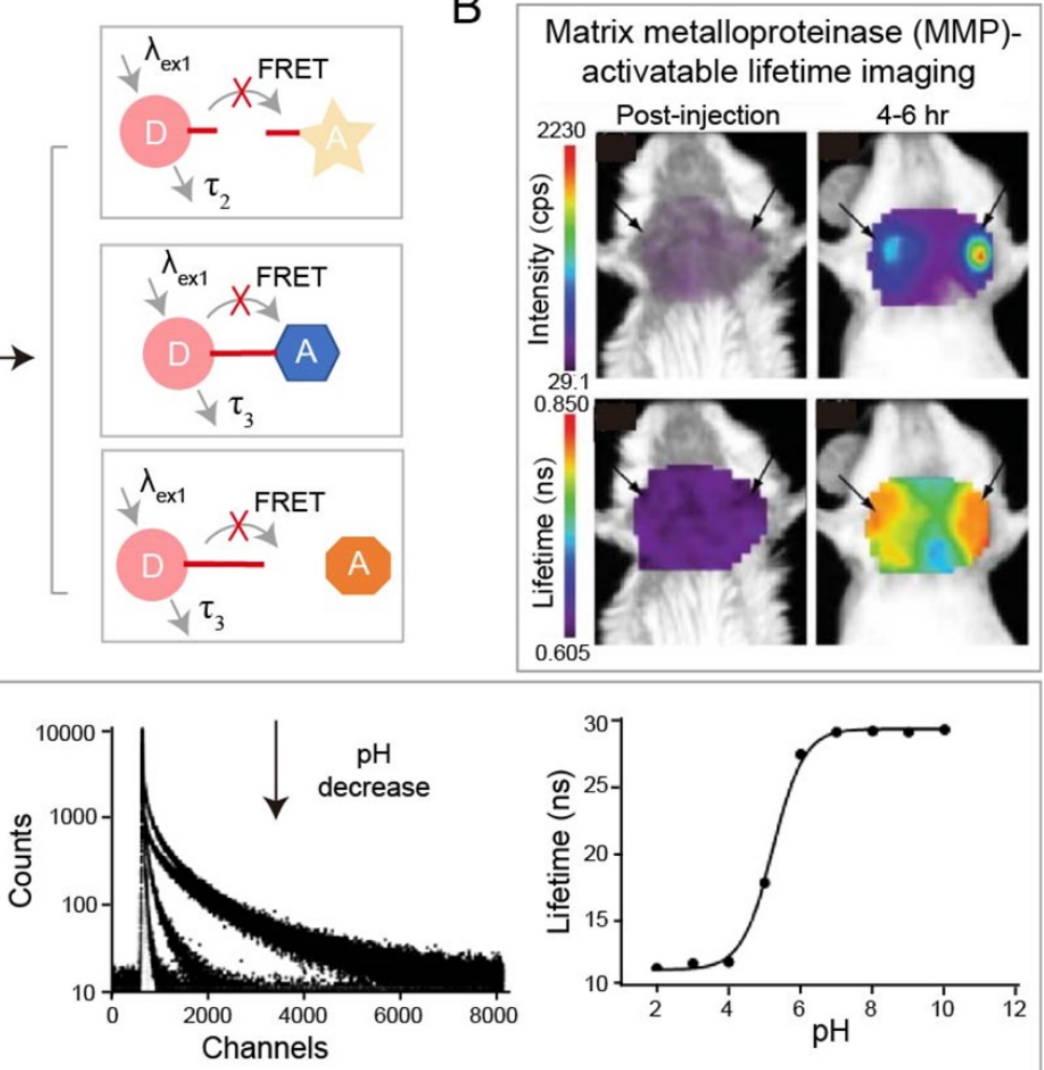

Figure 3. (A) Schematic of FRET-based luminescence probe and restoration of luminescence after stimulation by enzyme, $\mathrm{H}^{+}$, ROS, RNS etc. for lifetime imaging. (B) Luminescence imaging and luminescence lifetime imaging of breast tumors after injection of MMP750 (ex/em: 749/775 nm). Adapted with permission from [85], copyright 2011. (C) Illustration of FRET system of CdTeSe/ZnS quantum dots (QDs) and pH-sensitive organic dyes (left). Luminescence lifetime decay profile of the QD-dye system at different $\mathrm{pH}$ values (middle). Fit of the Luminescence lifetime vs $\mathrm{pH}$ (right). Adapted with permission from [74], copyright 2012.

\section{Activatable luminescence lifetime imaging}

The luminescence lifetime variation of probes responding to stimuli is the basis for a wide range of sensing applications. Förster resonance energy transfer (FRET) technique is the most common strategy for specific response to biological events both in vitro and in vivo. Adjacent dyes can interfere with the residence time of dyes in the excited state, resulting in a decrease of the mean residence time of dye in the excited state. In FRET system, luminescence lifetime of the energy donor decreases due to the energy transfer from energy donor to acceptor. After being activated by stimuli including $\mathrm{H}^{+}$, reactive Oxygen Species (ROS), reactive nitrogen species (RNS), lifetime recovery could be observed, which could be used for in vitro and in vivo detection (Figure 3A). This process will happen when the acceptor and donor are the identical dyes (homoFRET) or the different dyes (heteroFRET). When the dye is covalently attached to a polymer or biological macromolecules, the absorption and emission spectra will change, in most cases, the luminescence of dye will be quenched. Except the variation in luminescence intensity, the variation of lifetime can also be used to detect biological activity. NIR-I dyes, QDs and RENPs have been used to design FRET system with excellent luminescence lifetime biosensing. Solomon and coworkers reported a selfquenched matrix metalloproteinase (MMP)-activatable FRET probe using MMPSense750 (ex/em: 749/775 $\mathrm{nm}$ ), which is used for a murine orthotopic breast cancer model through both luminescence intensity and lifetime imaging (Figure 3B) [85]. This probe showed significant increases in luminescence intensity (10-fold) and lifetime (1.2-fold) at 4-6 h post-injection, suggesting the upregulated $\mathrm{MMP}$ activity in tumor tissue. Goergen and coworkers employed enzyme activatable probe, PGC-800 (ex/em: 778/794 nm), for imaging the expression of cathepsin B in mouse infarcted myocardium [86]. Due to the cleavage of specific amide bonds by cathepsin $\mathrm{B}$, the lifetime of probe recovered from $0.29 \pm 0.01$ to $0.47 \pm 0.01$ ns. Most importantly, luminescence lifetime imaging can distinguish the cathepsin B overexpressed infarct area from normal liver. Meanwhile, Alford and coworkers reported a self-quenched probe that is composed of antibody 
trastuzumab (targeting human epidermal growth factor receptor-2, HER2) conjugated to Alexa Fluor750 with different ratios of 1:8 or 1:1 (ex/em: $749 / 775 \mathrm{~nm}$ ) for monitoring cellular internalization by luminescence intensity and lifetime imaging. These results proved that HER2 cells can be monitored using tumor-specific activatable lifetime probe [97]. Achilefu and coworkers designed a $\mathrm{pH}$ sensing FRET nanomaterial of CdTeSe/ZnS QDs and LS662 (Figure 3C) [74]. The nanomaterials transfer the $\mathrm{pH}$ sensitivity of LS662 to long-lifetime QDs, thereby inducing a reversible luminescence lifetime variation between 29 ns at $\mathrm{pH}>7$ and $12 \mathrm{~ns}$ at $\mathrm{pH}<5$. Recently, Li and coworkers reported a FRET nanocomposite consisting of $\mathrm{Tm}^{3+}$-doped RENPs (as donor) and IR-820 (as acceptor) for luminescence lifetime imaging [87]. In the presence of $\mathrm{ClO}^{-}$, IR-820 would be destroyed, leading to luminescence lifetime recovery of RENPs from $200 \mu$ s to $600 \mu$ s at $800 \mathrm{~nm}$. The $\mathrm{ClO}^{-}$responsive luminescence lifetime imaging was successfully performed in a mouse arthritis model.

Many oxygen-containing or nitrogen-containing organic dyes have protonated and deprotonated forms, leading to the variation in their luminescence intensity or emission wavelength at different $\mathrm{pH}$ values. The luminescence lifetime would also change along with the molecular luminescence states during the protonation or deprotonation processes. Thus, by measuring $\mathrm{pH}$-induced changes in luminescence lifetime, the mapping of $\mathrm{pH}$ in cells and tissues can be obtained. Almutairi and coworkers have developed $\mathrm{pH}$-sensing biodegradable NIR-I nanoparticles (ex/em: 773/820 nm) for luminescence intensity and lifetime imaging (Figure 4A) [76]. Under neutral and alkaline environments, the NIR-I dye in the nanoparticles exists in the form of aggregates with weak luminescence and short lifetime. Under the acidic condition, nanoparticles would be broken and the released NIR-I dye bind to bovine serum albumin (BSA), resulting in increased luminescence intensity and lifetime (from 0.36 to $0.98 \mathrm{~ns}$ ). Berezin and coworkers have also reported NIR-I pH-sensing luminescence lifetime molecular probe, LS482 (ex/em: $700 / 780 \mathrm{~nm}$ ), with pKa $\sim 5.5$ for biological application (Figure 4B) [88]. LS482 has shown excellent $\mathrm{pH}$ sensitivity lifetime change with two different molecular forms, acidic ( 1.16 ns) and basic forms ( 1.4 ns). Then, when the LS482 probe is administrated subcutaneously, the luminescence lifetimes signal exhibited robust values and are not influenced by tissue penetration. These results demonstrated the feasibility of $\mathrm{pH}$ sensing nanoparticles for luminescence imaging in animals.

A

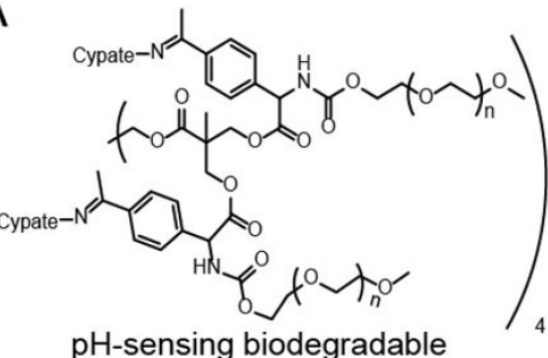

NIR-I nanoparticles

Ex/Em: 773 / 820 nm

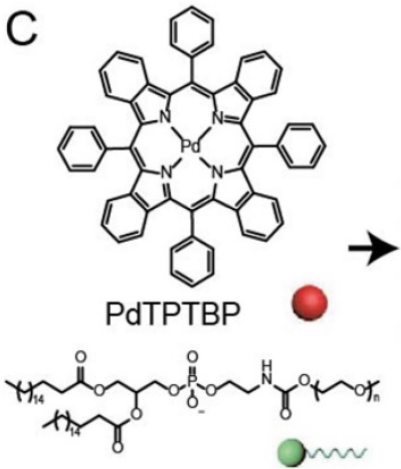

DSPE-PEG2000
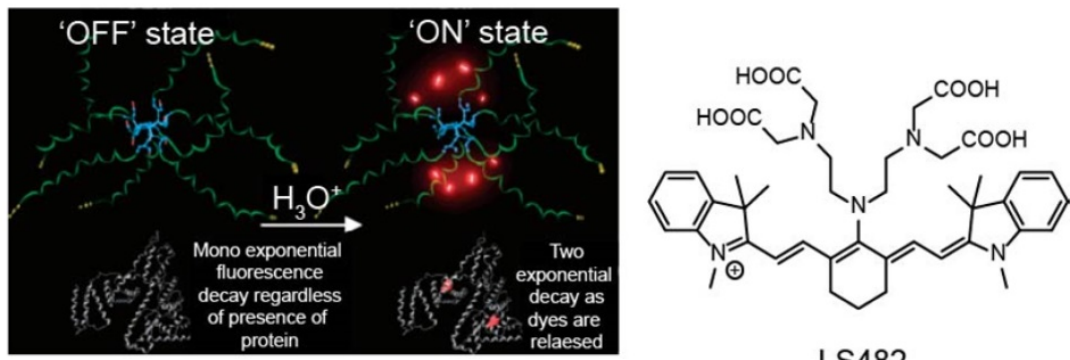

LS482

Ex/Em: $700 / 780 \mathrm{~nm}$

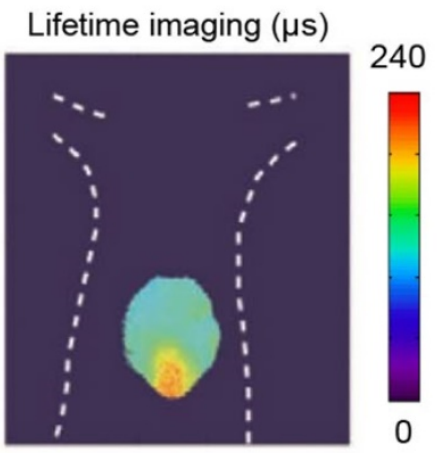

Figure 4. (A) Chemical structure of pH-sensing biodegradable NIR-I nanoparticles (ex/em: 773/820 nm) (left). The mechanism of pH sensing nanoparticles, "OFF: state: fluorescence lifetime of the nanoprobe is silenced in the neutral $\mathrm{pH}$ condition; "ON" state: fluorescence lifetime is increased when it binds to proteins in the acidic $\mathrm{pH}$ condition(right). Adapted with permission from [88], copyright 2008. (B) Chemical structure of pH probe LS482 (ex/em: $700 / 780 \mathrm{~nm}$ ). (C) Illustration of synthesis of nanoparticles Pd-MX (left). Luminescence lifetime decay profile of the Pd-MX at different oxygen levels (middle). Luminescence imaging of liver tumors at $12 \mathrm{~h}$ after injection of Pd-MX (ex/em: 635/800 nm) (right). Adapted with permission from [90], copyright 2020. 
Oxygen is an efficient luminescence quencher for almost all fluorophores due to collisional quenching. Strong quenching by oxygen is valuable for probes according to the decreased intensity or lifetime. Therefore, luminescence lifetime fluorophores, such as phosphorescent metal-ligand complexes (MLCs), are suitable for monitor tissue oxygenation. For example, Schreml and coworkers reported a palladium complex (ex/em: 444/797 nm) for imaging oxygen distribution and tissue hypoxia [89]. Recently, Liu and coworkers have been developed an oxygen sensitive nanoparticle, Pd-MX (ex/em: 635/800 nm), containing palladium complex for quantitative mapping the distribution of oxygen in hypoxia hepatic tissue and hepatic tumor lesion in mouse via phosphorescence lifetime imaging (Figure 4C) [90]. Additionally, researchers have also developed luminescence lifetime probes for detecting specific molecules, such as hydrogen peroxide and nitric oxide. Lifetime imaging of cells or tissues for these species is still evolving, and the development of molecular probes will help understand the physiological changes of related analytes.

\section{Luminescence lifetime imaging in NIR-II window}

Compared with NIR-I region, photons in NIR-II region have deeper tissue penetration due to the reduced tissue scattering and absorbance $[59,98]$. Thus, luminescence lifetime imaging in NIR-II region has more advantages in large animal models. Up to now, only rare-earth doped nanoparticles (RENPs) are used as contrast agents for NIR-II luminescence lifetime imaging due to the long and adjustable lifetime from microseconds to milliseconds. Similar to the classification criteria of NIR-I, luminescence lifetime imaging in NIR-II region can also be classified as non-activatable and activatable luminescence lifetime imaging.

\section{Non-activatable luminescence lifetime imaging in NIR-II window}

Due to tissue absorption and scattering of excitation and emission light, inhomogeneous signal attenuation occurs inevitably during luminescence bioimaging, which is the main problem for quantitative detection during multiplexed imaging. Luminescence lifetime that does not change with tissue penetration depth and probe concentration holds great value. Therefore, luminescence lifetime imaging is suitable for multiplexed imaging to observe the distribution of probes with different lifetimes in organ and tissue of interest. Zhang and coworkers have reported a general method based on controlled energy relay for RENPs to regulate their
NIR-II luminescence lifetimes. Adjustable lifetimes can be achieved universally in NIR-II region by selecting various emitting ions, including $\mathrm{Nd}^{3+}$ at 1060 $\mathrm{nm}, \mathrm{Ho}^{3+}$ at $1155 \mathrm{~nm}, \mathrm{Pr}^{3+}$ at $1289 \mathrm{~nm}, \mathrm{Tm}^{3+}$ at $1475 \mathrm{~nm}$ and $\mathrm{Er}^{3+}$ at $1525 \mathrm{~nm}$ [61]. For example, $\mathrm{Er}^{3+}$-doped RENPs has a wide range of luminescence lifetime from 1.25 to $7.21 \mathrm{~ms}$, which can be accommodate 11 distinct lifetime identities. Three kinds of $\mathrm{Er}^{3+}$-doped RENPs with different lifetimes were conjugated with three different antibodies to target estrogen receptor (ER), progesterone receptor (PR) and human epidermal growth factor receptor-2 (HER2) on breast cancer cells, which successfully achieved multiplexed imaging to distinguish different subtypes of breast tumor. These results demonstrated that multiplexed lifetime imaging could be used as a minimally invasive approach for disease diagnosis. Unfortunately, the reported RENPs have low quantum yields (0.009-0.235\%), which leads to long data acquisition time and cannot realize rapid lifetime imaging.

\section{Activatable luminescence lifetime imaging in NIR-II window}

Thanks to deep tissue penetration of NIR-II imaging and reliability of lifetime imaging, activatable NIR-II lifetime imaging has great potential in biological detection. For example, an inorganicorganic hybrid probe has successfully achieved a corresponding lifetime change to detect biomolecules. Zhang and coworkers have reported NIR-II luminescence lifetime FRET sensor DSNP@MY-1057GPC-3 for hepatocellular carcinoma (HCC) detection and quantitative ONOO- sensing (Figure 5A) [54]. $\mathrm{Nd}^{3+}$-doped RENPs with $1060 \mathrm{~nm}$ emission acted as FRET donor, and NIR-II absorption dye MY-1057 was chosen as FRET acceptor. Upon addition of ONOO-, a kind of reactive nitrogen species (RNS) in tumor, MY-1057 would be gradually decomposed, leading to lifetime recovery from $203 \pm 2$ to $298 \pm 2 \mu$ s (Figure 5B). Compared with intensity-based imaging, luminescence lifetime-based detection was not influenced by tissue penetration depth, leading to a high reliablilty during quantitative in vivo ONOOdetection (Figure 5C). These results indicated that lifetime imaging can quantitatively detect analytes under unknown tissue penetration depth. Due to excellent characteristics of lifetime imaging, multi-HCC lesions with lifetimes ranging from $215 \pm$ 27 to $249 \pm 43 \mu \mathrm{s}$ were easily distinguished from normal tissues which had a lifetime of $205 \pm 7 \mu \mathrm{s}$, while tumor lesions could not be identified by intensity-based imaging. Meanwhile, lifetime imaging, MRI and dissected imaging results were consistent, indicating the accuracy and reliability of 
lifetime imaging (Figure 5D). Due to the high stability and reliability, luminescence lifetime imaging is a promising and powerful imaging modality to provide qualitative and quantitative analysis ability for in vivo biosensing and bioimaging.

\section{Summary and Outlook}

Luminescence intensity imaging will continue to be widely used in conventional bio-imaging and bio-sensing. Yet, luminescence lifetime imaging is a new technology, which has been rapidly developing in recent years. As the intrinsic property of luminescent contrast agents, lifetime imaging can provide more information that may not be obtained from intensity-based imaging. The independence of luminescence lifetime on the materials concentration, intensity and tissue penetration depth overcomes spectral overlap and background interference in wavelength or intensity dependent multiplexed imaging and detection. Therefore, lifetime imaging could not only perform qualitative, but also achieve quantitative analysis. Recently, the trend towards deeper tissue imaging with NIR light, especially NIR-II, has received extensive attention. Wide-field NIR-II luminescence lifetime imaging has been used for in vivo multiplexed imaging and bio-sensing, which provides a good basis for subsequent clinical applications.
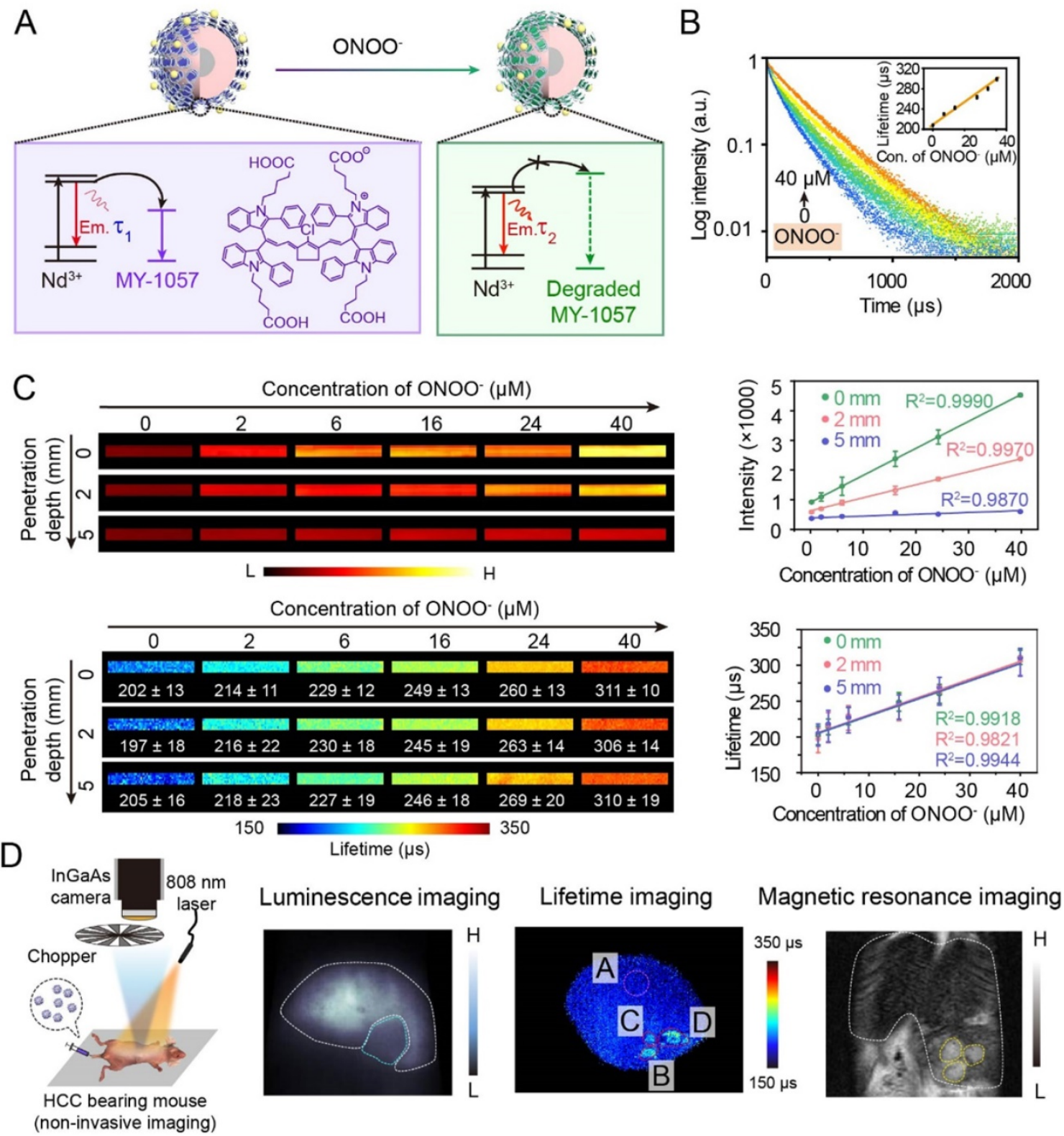

Figure 5. (A) Illustration of luminescence lifetime FRET sensor DSNP@MY-1057-GPC-3 for ONOO- and hepatocellular carcinoma (HCC) detection in NIR-II region. (B) Luminescence lifetime decay profile of sensor at different concentration of ONOO-. (C) Luminescence intensity and lifetime imaging of sensor at different penetration depths (1\% Intralipid) with increased ONOO- concentration. (D) Experimental setup used to for NIR-II lifetime imaging (right side). NIR-II luminescence intensity imaging, luminescence lifetime imaging and magnetic resonance imaging (MRI) of HCC tumor. ROI A: healthy hepatic tissue; B-D: tumor lesions identified by lifetime imaging. Adapted with permission from [54], copyright 2020. 
Despite some progress, there are still many challenges to be addressed to further promote bioimaging and biosensing in this promising field. (1) The number and types of materials required for luminescence lifetime imaging are relatively small. (2) The design concept of luminescence lifetime activatable materials is relatively simple. (3) There are few reports on the detection of biomarkers or biomolecules by lifetime imaging. (4) Luminescence lifetime imaging instrumentation is expensive, and lifetime data acquisition is generally slow. In order to promote the development of luminescence lifetime imaging, several directions needed to be considered in future studies:

\section{Developing more luminescence lifetime probes with high stability and quantum yields}

At present, only a small number and a few types of materials used for NIR luminescence lifetime imaging have been reported, including a few molecular dyes, transition metal-based complexes, and RENPs. In the next step, researchers may try to use other types of materials, such as QDs, SWCNTs, SPN etc. for lifetime imaging. The current materials for NIR activatable luminescence lifetime imaging mainly contain activatable molecules, transition metal-based complexes, and FRET nano-composites. As for NIR activatable luminescence lifetime materials based on FRET system, RENPs with long lifetime from $\mu$ s to $\mathrm{ms}$ are suitable as donors. And the reported NIR activatable acceptors of FRET nano-composites are organic molecules. However, difficulties in designing and synthesizing FRET acceptors hinder the construction of FRET nano-composites, especially in NIR-II region. To solve this problem, researcher could develop more types of acceptors of FRET nano-composites. The performance of the lifetime probes, including high quantum yields and high stability (chemical stability and photostability), are the key factors for luminescence lifetime imaging. Higher quantum yield brings faster imaging speeds and less administration dosage. Higher stability can avoid false signal results. Unfortunately, most of the reported NIR lifetime probes have low quantum yields and poor stability. As for organic dyes, rational design strategies, including changing terminal groups, introduction rigid plane chemical structure can increase the stability and improve the quantum yields. As for RENPs, adjusting the type of doped ions and designing new structures also can achieve above goal.

\section{Developing more generic lifetime activatable design strategy}

Activatable materials based on changes in luminescence intensity have been widely used for detecting or imaging specific biomarkers or physiological processes in living system. There are many reasons for the luminescence intensity change of the material, such as the change of the molecular aggregation state, and the energy transfer between luminescence materials and quenchers. This process generally leads to the change of the lifetime. Therefore, the traditional idea of designing luminescence intensity changes, such as FRET, intramolecular charge transfer (ICT), photoinduced electron transfer (PET), can be used to design activatable lifetime probes. Meanwhile, through these design strategies, more lifetime activatable probes can be developed to detect more biomolecules, such as enzyme, metal ions. In addition, another thing to note is that the variation range of the activatable lifetime of probe should be wide enough to provide high sensitivity and accuracy during detection.

\section{Activatable luminescence lifetime probes with multiplexed activation}

The occurrence and development of diseases are generally accompanied by changes in multiple physiological indicators. Compared with current single-parameter activatable lifetime probes, lifetime probes which can simultaneously detect the changes of multiple physiological indexes, will significantly improve the accuracy of disease detection. This will be one of the future directions of activatable luminescence lifetime imaging.

\section{Combination of luminescence lifetime and theranostics}

At present, a large amount of basic research works focused on simple luminescence lifetime without further consideration of therapy, which wastes the therapeutic potential of probes. By exploring the therapeutic potential of the lifetime probe itself, it can realize the diagnosis and treatment of diseases or observe the process of physiological changes under the guidance of luminescence lifetime imaging. Different types of lifetime probes have different optical and chemical functions. It is a good choice to further explore its diagnostic and therapeutic potential with minimal side effects and maximum therapeutic effects. In addition, the integration of diagnosis and therapy can also be achieved through simple loading or conjugated with drug.

\section{Developing luminescence lifetime imaging instrument}

Progress in luminescence lifetime platforms strongly depends on imaging equipment for 
multiplexed imaging or quantitative detection. However, lifetime imaging instrumentation is more costly than intensity or wavelength measurements. In addition, lifetime data acquisition speed is generally slower than intensity imaging. This is mainly because the fluorescence lifetime of each pixel on the detector requires more photons to be collected and accurately calculated. This makes it impossible to monitor and image rapidly occurring biological processes. So far, the commonly used luminescence lifetime imaging system is based on a data acquisition card and an optical chopper to control time synchronization. The rotation speed of the optical chopper and the response time of the transistor-transistor logic modulated laser are the factors affecting the lifetime imaging speed. Meanwhile, introducing more than two pieces of achromatic lens into imaging system can cause the efficiency decrease of fluorescence collection. Therefore, the ideal imaging instrument should have short data acquisition and analysis time, high temporal-spatial resolution for both cellular and deep tissue imaging. In order to achieve the above goals, equipment modification and software upgrade are the main concerns. Developing more advanced algorithms and imaging systems, or using advanced computers can speed up data acquisition and processing.

\section{Clinical translation of luminescence lifetime imaging}

Excellent probes with good biocompatibility, high quantum yields and stability, inexpensive equipment, and software upgrades are essential prerequisites for clinical translation. Lifetime imaging has almost no background interference, which is of great significance for clinical applications. First, the lifetime kits can be used to detect in vitro samples, such as tissues, blood, saliva, urine, feces, etc. Lifetime probes kits which can simultaneously detect multiple markers for a disease, can significantly improve the accuracy of disease detection. The results of the life test can be used as a supplement to the conclusions drawn by current clinical testing methods. Then, with the development of science and technology, lifetime-based imaging, and diagnostics, have been achieved at small animals' level, such as mice. Large animal measurement, even human-level measurement may also be realized if researchers could overcome the above-mentioned issues including probe and equipment development. For in vivo clinical applications, lifetime imaging can be used to detect some shallow tissue like skin diseases. Meanwhile, the development of lifetime endoscope imaging for deep tissue is also the direction of future development.

\section{Abbreviations}

BSA: bovine serum albumin; DTTCI: 3,3-diethylthiatricarbocyanine iodine; ER: estrogen receptor; FRET: Förster resonance energy transfer; HER2: human epidermal growth factor receptor-2; HCC: hepatocellular carcinoma; ICT: intramolecular charge transfer; MMP: matrix metalloproteinase; MRI: magnetic resonance imaging; MLCs: metal-ligand complexes; NIR: near-infrared; NIR-II: second near-infrared window; PR: progesterone receptor; QDs: quantum dots; SWCNTs: single-walled carbon nanotubes; SPN: semiconducting polymer nanomaterials; SWIR: shortwave infrared region; TCSPC: time correlated single photon counting; PET: photoinduced electron transfer; RENPs: rare-earth doped nanoparticles; ROS: reactive Oxygen Species; RNS: reactive nitrogen species.

\section{Acknowledgments}

The work is financially supported by National Key R\&D Program of China (2020YFA0908800, 2018YFA0704000), Basic Research Program of Shenzhen (JCYJ20180507182413022, JCYJ20170412111100 742), Guangdong Province Natural Science Foundation of Major Basic Research and Cultivation Project (2018B030308003), Shenzhen Science and Technology Program (KQTD20190929172538530), the National University of Singapore Start-up Grant (NUHSRO/2020/133/Startup/08) and NUS School of Medicine Nanomedicine Translational Research Programme (NUHSRO/2021/034/TRP/09/Nanomedicine).

\section{Competing Interests}

The authors have declared that no competing interest exists.

\section{References}

1. Falati S, Gross P, Merrill-Skoloff G, Furie BC, Furie B. Real-time in vivo imaging of platelets, tissue factor and fibrin during arterial thrombus formation in the mouse. Nat Med. 2002; 8: 1175-80.

2. Ntziachristos V, Ripoll J, Wang LV, Weissleder R. Looking and listening to light: the evolution of whole-body photonic imaging. Nat Biotechnol. 2005; 23: 313-20.

3. Horton NG, Wang K, Kobat D, Clark CG, Wise FW, Schaffer CB, et al. In vivo three-photon microscopy of subcortical structures within an intact mouse brain. Nat Photonics. 2013; 7: 205-09.

4. Zhang X, Tian Y, Zhang C, Tian X, Ross AW, Moir RD, et al. Near-infrared fluorescence molecular imaging of amyloid beta species and monitoring therapy in animal models of Alzheimer's disease. Proc Natl Acad Sci USA. 2015; 112: 9734-39.

5. Wan H, Du H, Wang F, Dai H. Molecular imaging in the second near-infrared window. Adv Funct Mater. 2019; 29: 1900566.

6. Li BH, Zhang YL, Li FS, Wang W, Liu J, Liu M, et al. A novel sensor for the detection of alkaline phosphatase activity based on the self-assembly of $\mathrm{Eu}^{3+}$-doped oxide nanoparticles and heptamethine cyanine dye. Sensor Actuat B: Chem. 2016; 233: 479-85.

7. Sun W, Guo S, Hu C, Fan J, Peng X. Recent development of chemosensors based on cyanine platforms. Chem Rev. 2016; 116: 7768-817.

8. Xu Y, Li B, Xiao L, Ouyang J, Sun S, Pang Y. A colorimetric and near-infrared fluorescent probe with high sensitivity and selectivity for acid phosphatase and inhibitor screening. Chem Commun. 2014; 50: 8677-80. 
9. $\mathrm{Li} \mathrm{B}, \mathrm{Li} \mathrm{W}, \mathrm{Xu} \mathrm{Y}, \mathrm{Li} \mathrm{J}, \mathrm{Tu}$ J, Sun S. A simple approach for the discrimination of surfactants based on the control of squaraine aggregation. Chem Commun. 2015; 51: 14652-55.

10. Gorka AP, Nani RR, Zhu J, Mackem S, Schnermann MJ. A near-IR uncaging strategy based on cyanine photochemistry. J Am Chem Soc. 2014; 136: 14153-59.

11. Cao W, Sletten EM. Fluorescent cyanine dye J-aggregates in the fluorous phase. J Am Chem Soc. 2018; 140: 2727-30.

12. Gu X, Zhang $P$, Wang J, Zheng $X$, Lam JWY, Tang BZ, et al. Corannulene-incorporated AIE nanodots with highly suppressed nonradiative decay for boosted cancer phototheranostics in vivo. Adv Mater. 2018; 30: 1801065 .

13. Zhang W, Huo F, Cheng F, Yin C. Employing an ICT-FRET integration platform for the real-time tracking of $\mathrm{SO} 2$ metabolism in cancer cells and tumor models. J Am Chem Soc. 2020; 142: 6324-31.

14. Gao X, Jiang S, Li C, Chen Y, Zhang Y, Huang P, et al. Highly photostable croconium dye-anchored cell membrane vesicle for tumor $\mathrm{pH}$-responsive duplex imaging-guided photothermal therapy. Biomaterials. 2021; 267: 120454

15. Zhao T, Huang G, Li Y, Yang S, Ramezani S, Lin Z, et al. A transistor-like pH nanoprobe for tumour detection and image-guided surgery. Nat Biomed Eng. 2016; 1: 0006.

16. He T, Yuan Y, Jiang C, Blum NT, He J, Lin J, et al. Light-triggered transformable ferrous ion delivery system for photothermal primed chemodynamic therapy. Angew Chem Int Ed Engl. 2020; 60: 6047-54.

17. Hu Z, Fang C, Li B, Zhang Z, Cao C, Cai M, et al. First-in-human liver-tumour surgery guided by multispectral fluorescence imaging in the visible and near-infrared-I/II windows. Nat Biomed Eng. 2020; 4: 259-71.

18. Xu Y, Li B, Han P, Sun S, Pang Y. Near-infrared fluorescent detection of glutathione via reaction-promoted assembly of squaraine-analyte adducts. Analyst. 2013; 138: 1004-07.

19. Sun Y, Ding F, Zhou Z, Li C, Pu M, Xu Y, et al. Rhomboidal Pt(II) metallacycle-based NIR-II theranostic nanoprobe for tumor diagnosis and imageguided therapy. Proc Natl Acad Sci USA. 2019; 116: 1968-73.

20. Xu Y, Li B, Li W, Zhao J, Sun S, Pang Y. "ICT-not-quenching" near infrared ratiometric fluorescent detection of picric acid in aqueous media. Chem Commun. 2013; 49: 4764-6.

21. Burda C, Chen XB, Narayanan R, El-Sayed MA. Chemistry and properties of nanocrystals of different shapes. Chem Rev. 2005; 105: 1025-102.

22. Medintz IL, Uyeda HT, Goldman ER, Mattoussi H. Quantum dot bioconjugates for imaging, labelling and sensing. Nat Mater. 2005; 4: 435-46.

23. Alivisatos AP. Semiconductor clusters, nanocrystals, and quantum dots. Science. 1996; 271: 933-37.

24. Michalet X, Pinaud FF, Bentolila LA, Tsay JM, Doose S, Li JJ, et al. Quantum dots for live cells, in vivo imaging, and diagnostics. Science. 2005; 307: 538-44.

25. Zhang Y, Hong G, Zhang Y, Chen G, Li F, Dai H, et al. Ag 2 S quantum dot: A bright and biocompatible fluorescent nanoprobe in the second near-infrared window. ACS Nano. 2012; 6: 3695-702.

26. Zhao M, Li B, Wang P, Lu L, Zhang Z, Liu L, et al. Supramolecularly engineered NIR-II and upconversion nanoparticles in vivo assembly and disassembly to improve bioimaging. Adv Mater. 2018; 30: 1804982

27. Naczynski D, Tan M, Zevon M, Wall B, Kohl J, Kulesa A, et al. Rare-earth-doped biological composites as in vivo shortwave infrared reporters. Nat Commun. 2013; 4: 1-10.

28. Zhong Y, Ma Z, Zhu S, Yue J, Zhang M, Antaris AL, et al. Boosting the down-shifting luminescence of rare-earth nanocrystals for biological imaging beyond $1500 \mathrm{~nm}$. Nat Commun. 2017; 8: 737

29. Wang F, Liu X. Recent advances in the chemistry of lanthanide-doped upconversion nanocrystals. Chem Soc Rev. 2009; 38: 976-89.

30. Dong B, Cao B, He Y, Liu Z, Li Z, Feng Z. Temperature sensing and in vivo imaging by molybdenum sensitized visible upconversion luminescence of rare-earth oxides. Adv Mater. 2012; 24: 1987-93.

31. Zhong Y, Ma Z, Wang F, Wang X, Yang Y, Liu Y, et al. In vivo molecular imaging for immunotherapy using ultra-bright near-infrared-Ilb rare-earth nanoparticles. Nat Biotechnol. 2019; 37: 1322-31.

32. Welsher K, Liu Z, Sherlock SP, Robinson JT, Chen Z, Daranciang D, et al. A route to brightly fluorescent carbon nanotubes for near-infrared imaging in mice. Nat Nanotechnol. 2009; 4: 773-80.

33. Ghosh D, Bagley AF, Na YJ, Birrer MJ, Bhatia SN, Belcher AM. Deep, noninvasive imaging and surgical guidance of submillimeter tumors using targeted M13-stabilized single-walled carbon nanotubes. Proc Natl Acad Sci USA. 2014; 111: 13948-53

34. Ning Y, Cheng S, Wang J-X, Liu Y-W, Feng W, Li F, et al. Fluorescence lifetime imaging of upper gastrointestinal $\mathrm{pH}$ in vivo with a lanthanide based near-infrared t probe. Chem Sci. 2019; 10: 4227-35.

35. Zhang T, Zhu X, Cheng CC, Kwok WM, Tam HL, Hao J, et al. Water-soluble mitochondria-specific ytterbium complex with impressive NIR emission. J Am Chem Soc. 2011; 133: 20120-2

36. Li J, Pu K. Development of organic semiconducting materials for deep-tissue optical imaging, phototherapy and photoactivation. Chem Soc Rev. 2019; 48: 38-71.

37. Gong X-T, Xie W, Cao J-J, Zhang $\mathrm{S}, \mathrm{Pu} \mathrm{K}$, Zhang H-L. NIR-emitting semiconducting polymer nanoparticles for in vivo two-photon vascular imaging. Biomater Sci. 2020; 8: 2666-72.
38. Li B, Zhao M, Feng L, Dou C, Ding S, Zhou G, et al. Organic NIR-II molecule with long blood half-life for in vivo dynamic vascular imaging. Nat Commun. 2020; $11: 3102$

39. Tian R, Zhu S, Lau J, Chandra S, Niu G, Kiesewetter DO, et al. Albumin-chaperoned cyanine dye yields superbright NIR-II fluorophore with enhanced pharmacokinetics. Sci Adv. 2019; 5: eaaw067.

40. Li B, Lu L, Zhao M, Lei Z, Zhang F. An efficient $1064 \mathrm{~nm}$ NIR-II excitation fluorescent molecular dye for deep-tissue high-resolution dynamic bioimaging. Angew Chem Int Ed Engl. 2018; 57: 7483-87.

41. Zhao M, Wang R, Li B, Fan Y, Wu Y, Zhu X, et al. Precise in vivo inflammation imaging using in situ responsive cross-linking of glutathione-modified ultra-small NIR-II lanthanide nanoparticles. Angew Chem Int Ed Engl. 2019; 58: 2050-54.

42. Zeng X, Xiao Y, Lin J, Li S, Zhou H, Nong J, et al. Near-infrared II dye-protein complex for biomedical imaging and imaging-guided photothermal therapy. Adv Healthc Mater. 2018; 7: 1800589.

43. Bruns OT, Bischof TS, Harris DK, Franke D, Shi Y, Riedemann L, et al. Next-generation in vivo optical imaging with short-wave infrared quantum dots. Nat Biomed Eng. 2017; 1: 56.

44. Zhao M, Li B, Fan Y, Zhang F. In vivo assembly and disassembly of probes to improve near-infrared optical bioimaging. Adv Healthc Mater. 2019; 8: 1801650.

45. Zhu S, Hu Z, Tian R, Yung BC, Yang Q, Zhao S, et al. Repurposing cyanine NIR-I dyes accelerates clinical translation of near-infrared-II (NIR-II) bioimaging. Adv Mater. 2018; 30: 1802546.

46. Zhu S, Yung BC, Chandra S, Niu G, Antaris AL, Chen X. Near-infrared-II (NIR-II) bioimaging via off-peak NIR-I fluorescence emission. Theranostics. 2018; 8: 4141.

47. Carr JA, Franke D, Caram JR, Perkinson CF, Saif M, Bawendi MG, et al. Shortwave infrared fluorescence imaging with the clinically approved near-infrared dye indocyanine green. Proc Natl Acad Sci USA. 2018; 115: 4465-70.

48. Antaris AL, Chen H, Sun Y, Qu C, Deng Z, Hu X, et al. A small-molecule dye for NIR-II imaging. Nat Mater. 2016; 15: 235-42

49. Chen W, Cheng CA, Cosco ED, Ramakrishnan S, Lingg JGP, Bruns OT, et al. Shortwave infrared imaging with J-aggregates stabilized in hollow mesoporous silica nanoparticles. J Am Chem Soc. 2019; 141: 12475-80.

50. Sun Y, Zeng X, Xiao Y, Liu C, Zhu H, Zhou $\mathrm{H}$, et al. Novel dual-function near-infrared II fluorescence and PET probe for tumor delineation and image-guided surgery. Chem Sci. 2018; 9: 2092-97.

51. Li C, Li W, Liu H, Zhang Y, Chen G, Li Z, et al. An activatable NIR-II nanoprobe for in vivo early real-time diagnosis of traumatic brain injury. Angew Chem Int Ed Engl. 2020; 59: 247-52.

52. Wang S, Li B, Zhang F. Molecular fluorophores for deep-tissue bioimaging. ACS Cent Sci. 2020; 6: 1302-16.

53. Zhao M, Wang J, Lei Z, Lu L, Wang S, Zhang H, et al. NIR-II pH sensor with a FRET adjustable transition point for in situ dynamic tumor microenvironment visualization. Angew Chem Int Ed Engl. 2021; 60: 5091-95.

54. Zhao $\mathrm{M}$, Li B, Wu Y, He H, Zhu $\mathrm{X}$, Zhang $\mathrm{H}$, et al. A tumor-microenvironment-responsive lanthanide-cyanine FRET sensor for NIR-II luminescence-lifetime in situ imaging of hepatocellular carcinoma. Adv Mater. 2020; 32: 2001172

55. Ma Z, Zhang M, Yue J, Alcazar C, Zhong Y, Doyle TC, et al. Near-infrared IIb fluorescence imaging of vascular regeneration with dynamic tissue perfusion measurement and high spatial resolution. Adv Funct Mater. 2018; 28: 1803417.

56. Sun C, Li B, Zhao M, Wang S, Lei Z, Lu L, et al. J-aggregates of cyanine dye for NIR-II in vivo dynamic vascular imaging beyond $1500 \mathrm{~nm}$. J Am Chem Soc. 2019; 141: 19221-25.

57. Li B, Zhao M, Zhang F. Rational design of near-infrared-II organic molecular dyes for bioimaging and biosensing. ACS Mater Lett. 2020; 2: 905-17.

58. Li Y, Cai Z, Liu S, Zhang H, Wong STH, Lam JWY, et al. Design of AIEgens for near-infrared IIb imaging through structural modulation at molecular and morphological levels. Nat Commun. 2020; 11: 1255.

59. Zhao M, Li B, Zhang H, Zhang F. Activatable fluorescence sensors for in vivo bio-detection in the second near-infrared window. Chem Sci. 2021; 12: 344859

60. Zhou L, Fan Y, Wang R, Li X, Fan L, Zhang F. High-capacity upconversion wavelength and lifetime binary encoding for multiplexed biodetection. Angew Chem Int Ed Engl. 2018; 57: 12824-29.

61. Fan Y, Wang P, Lu Y, Wang R, Zhou L, Zheng X, et al. Lifetime-engineered NIR-II nanoparticles unlock multiplexed in vivo imaging. Nat Nanotechnol. 2018; 13: 941-46.

62. Becker W. Fluorescence lifetime imaging - techniques and applications. J Microsc. 2012; 247: 119-36.

63. Becker W. Fluorescence lifetime imaging by multi-dimensional time correlated single photon counting. Med Photon. 2015; 27: 41-61.

64. Borst JW, Visser A. Fluorescence lifetime imaging microscopy in life sciences. Meas Sci Technol Technol. 2010; 21: 102002.

65. Suhling K, Hirvonen LM, Levitt JA, Chung P-H, Tregidgo C, Le Marois A, et al. Fluorescence lifetime imaging (FLIM): Basic concepts and some recent developments. Med Photon. 2015; 27: 3-40

66. Berezin MY, Achilefu S. Fluorescence lifetime measurements and biological imaging. Chem Rev. 2010; 110: 2641-84.

67. Sarder P, Maji D, Achilefu S. Molecular probes for fluorescence lifetime imaging. Bioconjugate Chem. 2015; 26: 963-74. 
68. del Rosal B, Ortgies DH, Fernandez N, Sanz-Rodriguez F, Jaque D, Rodriguez EM. Overcoming autofluorescence: long-lifetime infrared nanoparticles for time-gated in vivo imaging. Adv Mater. 2016; 28: 10188-93.

69. Chen Y, Mills JD, Periasamy A. Protein localization in living cells and tissues using FRET and FLIM. Differentiation. 2003; 71: 528-41.

70. Chorvat D, Chorvatova A. Spectrally resolved time-correlated single photon counting: a novel approach for characterization of endogenous fluorescence in isolated cardiac myocytes. Eur Biophys J. 2006; 36: 73-83.

71. Cicchi R, Saverio Pavone F. Non-linear fluorescence lifetime imaging of biological tissues. Anal Bioanal Chem. 2011; 400: 2687-97.

72. Wilms CD, Eilers J. Photo-physical properties of $\mathrm{Ca}^{2+}$-indicator dyes suitable for two-photon fluorescence-lifetime recordings. J Microsc. 2007; 225: 209-13.

73. Paredes JM, Giron MD, Ruedas-Rama MJ, Orte A, Crovetto L, Talavera EM, et al. Real-Time phosphate sensing in living cells using fluorescence lifetime imaging microscopy (FLIM). J Phys Chem B. 2013; 117: 8143-49.

74. Tang R, Lee H, Achilefu S. Induction of $\mathrm{pH}$ sensitivity on the fluorescence lifetime of quantum dots by NIR fluorescent dyes. J Am Chem Soc. 2012; 134: 4545-48.

75. Ogikubo S, Nakabayashi T, Adachi T, Islam MS, Yoshizawa T, Kinjo M, et al. Intracellular $\mathrm{pH}$ sensing using autofluorescence lifetime microscopy. J Phys Chem B. 2011; 115: 10385-90.

76. Adah A, Steven J. G, Mikhail Y. Bn, Samuel A, F JMJ. Biodegradable $\mathrm{pH}$-sensing dendritic nanoprobes for near-infrared fluorescence lifetime and intensity imaging. J Am Chem Soc. 2008; 130: 444-45.

77. Benninger R, Koc Y, Hofmann O, Requejo-Isidro J, Neil M, French P, et al. Quantitative 3D mapping of fluidic temperatures within microchannel networks using fluorescence lifetime imaging. Anal Chem. 2006; 78: 2272-78,

78. Okabe K, Inada N, Gota C, Harada Y, Funatsu T, Uchiyama S. Intracellular temperature mapping with a fluorescent polymeric thermometer and fluorescence lifetime imaging microscopy. Nat Commun. 2012; 3: 705.

79. Datta R, Heaster TM, Sharick JT, Gillette AA, Skala MC. Fluorescence lifetime imaging microscopy: fundamentals and advances in instrumentation, analysis, and applications. J Biomed Opt. 2020; 25: 1-43.

80. Solomatina AI, Su S-H, Lukina MM, Dudenkova VV, Shcheslavskiy VI, Wu $\mathrm{C}-\mathrm{H}$, et al. Water-soluble cyclometalated platinum(II) and iridium(III) complexes: synthesis, tuning of the photophysical properties, and in vitro and in vivo phosphorescence lifetime imaging. RSC Adv. 2018; 8: 17224-36.

81. Yaseen MA, Sutin J, Wu W, Fu B, Uhlirova H, Devor A, et al. Fluorescence lifetime microscopy of NADH distinguishes alterations in cerebral metabolism in vivo. Biomed Opt Express. 2017; 8: 2368-85.

82. Nothdurft R, Sarder P, Bloch S, Culver J, Achilefu S. Fluorescence lifetime imaging microscopy using near-infrared contrast agents. J Microsc. 2012; 247: 202-7.

83. Ortgies $\mathrm{DH}, \mathrm{Tan} \mathrm{M}$, Ximendes $\mathrm{EC}$, del Rosal $\mathrm{B}, \mathrm{Hu} \mathrm{J}$, $\mathrm{Xu} \mathrm{L}$, et al. Lifetime-encoded infrared-emitting nanoparticles for in vivo multiplexed imaging. ACS Nano. 2018; 12: 4362-68.

84. Li H, Tan M, Wang X, Li F, Zhang Y, Zhao L, et al. Temporal multiplexed in vivo upconversion imaging. J Am Chem Soc. 2020; 142: 2023-30.

85. Solomon M, Guo K, Sudlow GP, Berezin MY, Edwards WB, Achilefu S, et al. Detection of enzyme activity in orthotopic murine breast cancer by fluorescence lifetime imaging using a fluorescence resonance energy transfer-based molecular probe. J Biomed Opt. 2011; 16: 066019.

86. Goergen CJ, Chen HH, Bogdanov A, Sosnovik DE, Kumar AT. In vivo fluorescence lifetime detection of an activatable probe in infarcted myocardium. J Biomed Opt. 2012; 17: 056001.

87. Kong M, Gu Y, Liu Y, Shi Y, Wu N, Feng W, et al. Luminescence lifetimebased in vivo detection with responsive rare earth-dye nanocomposite. Small. 2019; 15: 1904487

88. Berezin MY, Guo K, Akers W, Northdurft RE, Culver JP, Teng B, et al. Near-infrared fluorescence lifetime $\mathrm{pH}$-sensitive probes. Biophys J. 2011; 100: 2063-72.

89. Schreml S, Meier RJ, Kirschbaum M, Kong SC, Gehmert S, Felthaus O, et al Luminescent dual sensors reveal extracellular $\mathrm{pH}$-gradients and hypoxia on chronic wounds that disrupt epidermal repair. Theranostics. 2014; 4: 721-35.

90. Liu Y, Gu Y, Yuan W, Zhou X, Qiu X, Kong M, et al. Quantitative mapping of liver hypoxia in living mice using time-resolved wide-field phosphorescence lifetime imaging. Adv Sci. 2020; 7: 1902929

91. Gorris $\mathrm{HH}$, Wolfbeis OS. Photon-upconverting nanoparticles for optical encoding and multiplexing of cells, biomolecules, and microspheres. Angew Chem Int Ed Engl. 2013; 52: 3584-600.

92. Wilson R, Cossins AR, Spiller DG. Encoded microcarriers for high-throughput multiplexed detection. Angew Chem Int Ed Engl. 2006; 45: 6104-17.

93. Leng $\mathrm{Y}$, Sun $\mathrm{K}$, Chen $\mathrm{X}, \mathrm{Li}$ W. Suspension arrays based on nanoparticle-encoded microspheres for high-throughput multiplexed detection. Chem Soc Rev. 2015; 44: 5552-95.

94. Xu Y, Li B, Xiao L, Li W, Zhang C, Sun S, et al. The sphere-to-rod transition of squaraine-embedded micelles: a self-assembly platform displays a distinct response to cysteine and homocysteine. Chem Commun. 2013; 49: 7732-4.

95. Zhang Y, Zhang L, Deng R, Tian J, Zong Y, Jin D, et al. Multicolor barcoding in a single upconversion crystal. J Am Chem Soc. 2014; 136: 4893-96.

96. Zhu S, Yang Q, Antaris AL, Yue J, Ma Z, Wang H, et al. Molecular imaging of biological systems with a clickable dye in the broad $800-$ to $1,700-\mathrm{nm}$ near-infrared window. Proc Natl Acad Sci USA. 2017; 114: 962-67.
97. Alford R, Ogawa M, Hassan M, Gandjbakhche AH, Choyke PL, Kobayashi H. Fluorescence lifetime imaging of activatable target specific molecular probes. Contrast Media Mol Imaging. 2010; 5: 1-8.

98. Hong G, Antaris AL, Dai H. Near-infrared fluorophores for biomedical maging. Nat Biomed Eng. 2017; 1: 0010. 